\title{
Outage Probability of Device-to-Device Communications in Frequency Reuse-1 Networks
}

\author{
Marcin Rodziewicz $^{1}$ (D)
}

Published online: 8 February 2017

(C) The Author(s) 2017. This article is published with open access at Springerlink.com

\begin{abstract}
In this paper, we analyse the outage probability of Device-to-Device (D2D) communications in a multi-cell frequency reuse-1 (FRF-1) system. We derive a formula for outage probability of a D2D link in the FRF-1 system when uplink resource sharing between cellular users and D2D users is enabled in the network. Based on this analysis, we propose a location-based power control mechanism for D2D which can enhance the outage performance of the D2D link, and at the same time, reduce the transmit power required for communication.
\end{abstract}

Keywords Device-to-device $\cdot$ Reuse-1 - Outage probability $\cdot$ Power control

\section{Introduction}

In recent years Device-to-Device (D2D) underlay in cellular networks has attracted a lot of attention. It is believed that it can improve system spectral efficiency, reduce power consumption and enable a new type of multimedia services [1-3]. D2D communication was one of the main technologies that were thoroughly investigated within the UE FP7 METIS (Mobile and wireless communications Enablers for the Twenty-twenty Information Society) project [4]. Considering the D2D underlay, it is often assumed that D2D communications may use radio resources simultaneously with cellular users. This assumption allows for increasing

Marcin Rodziewicz

marcin.rodziewicz@put.poznan.pl

1 Faculty of Electronics and Telecommunication, Poznan University of Technology, Poznan, Poland the frequency reuse factor (FRF) even above the reuse-1 [3]. However, enabling D2D communications reusing the cellular spectrum poses new challenges, for instance, new interference patterns.

The frequency bandwidth is a scarce resource, thus the need to utilize it in the most efficient way. In this paper we analyse a D2D underlay reusing cellular uplink (UL) resources in frequency reuse-1 (FRF-1) network which aims at maximizing effectiveness of bandwidth use. We derive a formula for outage probability of a D2D link in a multi-cell FRF-1 system and propose a location-based power control mechanism for $\mathrm{D} 2 \mathrm{D}$ communication.

The remainder of the paper is organized as follows. Section 2 gives the background for the considerations of this paper. Section 3 describes the system model used in evaluation of the proposed concept and presents the closed-form expression for the outage probability of D2D communication reusing UL resources in a multi-cell FRF-1 environment. In Section 4 the outage probability analysis of D2D communication is contained. Section 4 also presents a proposal of location-based power control mechanism for D2D communication. The paper is concluded in Section 5.

\section{Related work}

The mitigation of interference inherent to D2D communications is a well studied topic [1, 2, 5-10] and the most common approaches involve power control and resource allocation solutions. For example, in [5] a D2D power reduction method was proposed to control the interference to cellular users (CUEs). With regards to interference mitigation solutions based on resource allocation many of the proposed methods (e.g. [8]) exploit the knowledge of the slow-scale parameters such as path-loss or shadowing 
to perform interference-aware resource allocation. Several solutions investigate the possibility of exploiting location information for resource allocation and sharing user selection [11-15]. In [11] and [12], apart from a power control mechanism, an interference limited area (ILA), also referred to as an interference limited ring (ILR), control strategy is proposed. The goal of this strategy is to guarantee that the outage probability of the D2D communication caused by the interference from CUEs is less than a predetermined threshold, while maintaining the interference level to CUEs at satisfactory level. On the other hand, in [13] the interference limited area control method is applied to restrict the interference prior to the resource allocation process. In [14], a distance-constrained resource-sharing criterion (DRC) is introduced which limits the set of CUEs that can share resources with considered D2D users (DUEs). The results show that DRC brings significant reduction of outage probability of the D2D link. Moreover, the proposed DRC does not require CUE to reduce its transmission power, and thus avoids degrading the performance of the cellular link. The work reported in [14] is further extended in [15], where the proposed user selection mechanism is evaluated in a multi-cell fractional frequency reuse (FFR) system. The authors evaluate the use of UL resource sharing for D2D along with strict FFR scheme in a three cell scenario. Strict FFR assumes that each cell is divided into inner and outer regions. Inner region of each cell uses the same frequency band e.g. A, whereas the outer regions use different bands e.g. B,C and D. In this approach the D2D communication is allowed to use only the inner region frequency band and the D2D pair has to be located within the outer region of the cell. The results of the three cell scenario show that the proposed resource sharing scheme can significantly improve the outage probability performance of the D2D link. Another paper that investigates the D2D communication in a FFR system is [16]. In this work a radio resource allocation is proposed where the DUEs and CUEs use different frequency bands that are chosen based on users' locations. The D2D communication in [16] takes place in the downlink (DL) and the result show that by using radio resources selectively (according to users' positions) can improve the total performance of the network.

All of the aforementioned papers assume either a singlecell scenario [1-3, 5-14] or a multi-cell scenario with FFR $[15,16]$. Single-cell considerations cannot be easily applied to systems exploiting frequency reuse due different interference patterns of such networks. In FRF-1 system the D2D underlay will experience interference from multiple sources as shown in Fig. 1. On the other hand FFR reduces the interference mitigation problem at the cost of less efficient bandwidth utilization compared to FRF-1 systems. Since the bandwidth is a scarce resource the use of D2D communications in FRF-1 system emerge as one of the solutions

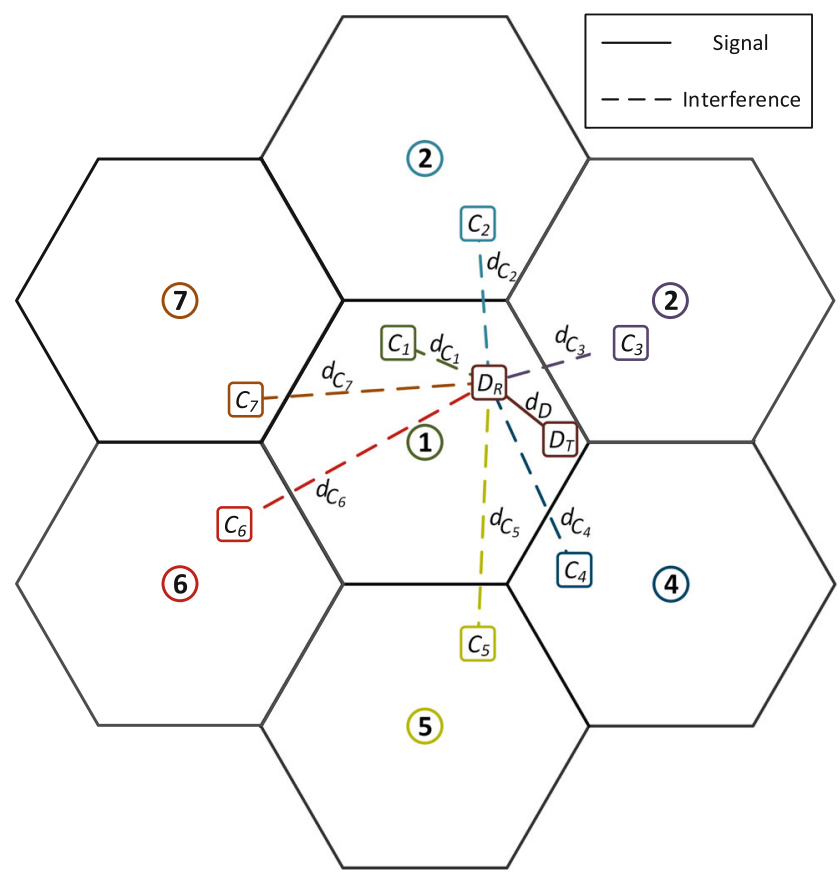

Fig. 1 Model of D2D communications underlaying a FRF-1 network

for more efficient bandwidth use. The main contribution of this paper is the analysis of the outage probability of a D2D communication in a multi-cell FRF-1 system. We derive a formula for outage probability of a D2D link in FRF-1 system when uplink resource sharing between CUEs and DUEs is enabled in the network. Based on this analysis, we propose a location-based power control mechanism for D2D which can enhance the outage performance of the D2D link, and at the same time, reduce the transmit power required for communication.

\section{System model}

We consider an OFDMA-based FRF-1 cellular network with a D2D underlay (Fig. 1). Base stations (BS) are located in central positions of hexagonal cells with edge length $R$ and inter-site distance (ISD) $S$. For any CUE, noted as $C_{i}$, its location is given in polar coordinates by a pair $\left(r_{C_{i}}, \varphi_{C_{i}}\right)$. The angle $\varphi_{C_{i}}$ follows the uniform distribution $[0,2 \pi)$ and the distance of the user to its serving BS is limited by cells' hexagonal shape, thus $r_{C_{i}}$ is a random variable with a probability density function (PDF):

$f_{r_{C_{i}}}\left(r_{C_{i}}\right)=\frac{6 r_{C_{i}} \sqrt{3}}{2 \pi R^{2}}$

where $r_{C_{i}} \in\left(0, S /\left|\sin \left(\varphi_{C_{i}}\right)\right|+\sqrt{3}\left|\cos \left(\varphi_{C_{i}}\right)\right|\right]$ for $\varphi_{C_{i}} \in$ $(-\pi / 3, \pi / 3) \cup(-2 \pi / 3,2 \pi / 3)$ and $r_{C_{i}} \in\left(0, S / 2\left|\sin \left(\varphi_{C_{i}}\right)\right|\right]$ for $\varphi_{C_{i}} \in(-\pi / 3,-2 \pi / 3) \cup(\pi / 3,2 \pi / 3)$. 
Apart from the cellular users, a D2D pair is deployed in the cell served by BS1. A D2D receiver $\left(D_{R}\right)$ is placed in the same manner as CUEs with location parameters $\left(r_{d}, \varphi_{d}\right)$, while a D2D transmitter $\left(D_{T}\right)$ is deployed on a circle of radius $d_{D}$ centred at the location of the $\mathrm{D} 2 \mathrm{D}$ receiver.

The D2D transmissions take place in the uplink resources of the cellular communication. As a result, a new interference pattern is introduced in the network. The $\mathrm{D} 2 \mathrm{D}$ receiver is a victim of interference caused by CUEs that share their resources with the D2D transmitter. On the other hand, the D2D transmitter generates additional interference towards CUEs. This interference is experienced by the CUEs serving BSs. In this paper we focus on the performance of the D2D link, thus we omit the impact of the D2D communication on cellular users under the assumption that the BS, with its strong processing capacity, is capable of mitigating the D2D-to-Cellular (D2C) interference.

Considering the geometry of the system shown in Fig. 1, and extending it to a generic $N$ cell case, the signal-to-noise and interference ratio (SINR) at the D2D receiver can be written as

$$
\gamma_{D_{R}}=\frac{\left|h_{D 2 D}\right|^{2} P_{D} d_{D}^{-\alpha}}{\sum_{n=1}^{N}\left|h_{n R}\right|^{2} P_{C_{n}} d_{C_{n}}^{-\alpha}+N_{0}},
$$

where $h_{D 2 D}$ and $h_{n R}$ are the fading coefficients of the D2D link and the link between the DUE receiver and the $n$ th CUE transmitter, respectively. Both follow the complex Gaussian distribution $\mathcal{C N}(0,1)$. The path-loss exponent is denoted with $\alpha$, whereas $N_{0}$ is the additive white Gaussian noise (AWGN) variance. The transmit power of the D2D transmitter and the $n$-th CUE are given by $P_{D}$ and $P_{C_{n}}$, respectively.

\subsection{Outage probability}

To analyse the performance of the D2D link in an FRF-1 network, we derive an expression for outage probability of the $\mathrm{D} 2 \mathrm{D}$ receiver, which is defined as the probability that instantaneous SINR $\gamma_{D_{R}}$ falls below a predetermined SINR threshold $\gamma_{0}$. The outage probability of a D2D receiver conditioned on the locations of the CUEs $\left(L_{C_{n}}\right)$ selected for resource sharing can be expressed as:

$\operatorname{Pr}\left[\gamma_{D_{R}}<\gamma_{0} \mid L_{C_{1}}, \ldots, L_{C_{n}}\right]=F_{\gamma_{D_{R}}}\left(\gamma_{0}\right)$,

where $F_{\gamma_{D_{R}}}(\cdot)$ is a cumulative distribution function (CDF) of $\gamma_{D_{R}}$. To obtain the outage probability of the D2D link, we use the following Lemma.

Lemma 1 Let $\left|h_{0}\right|^{2}$ follow an exponential distribution and $s=\sum_{m=1}^{M}\left|h_{m}\right|^{2} / \lambda_{m}+\sum_{l=1}^{L}\left|h_{l}\right|^{2} / \lambda_{e}$ be the sum of $N=$ $M+L$ exponential random variables, $M$ of which has a distinct mean $1 / \lambda_{m}$ and $L$ has the same mean $1 / \lambda_{e}$. Let $x=$ $\left|h_{0}\right|^{2} / \lambda_{0}, y=s+\delta$, and $\lambda_{0}, \lambda_{m}, \lambda_{e}, \delta>0$, then the $C D F$ of $z=x / y$ is:

$$
\begin{aligned}
F_{z}(z)= & \sum_{m=1}^{M} E_{m}\left(1-\frac{\lambda_{m} \exp \left(-\lambda_{0} z \delta\right)}{\lambda_{0} z+\lambda_{m}}\right) \\
& +\sum_{l=1}^{L} A_{l}\left[1-\frac{\lambda_{e}^{l} \exp \left(-\lambda_{0} z \delta\right)}{\left(\lambda_{0} z+\lambda_{e}\right)^{l}}\right] .
\end{aligned}
$$

The definition of $E_{m}$ and $A_{l}$ can be found in [17], whereas $\delta$ is a constant.

Proof With the help of [18, eq. (5-18)], the PDFs of $x$ and $y$ are expressed as $f_{x}(x)=\lambda_{0} \exp \left(-\lambda_{0} x\right) U(x)$ and $f_{y}(y)=$ $f_{s}(y-\delta) U(y-\delta)$, respectively. From [17] the PDF of $f_{s}(s)$ is:

$$
\begin{aligned}
f_{s}(s)= & \sum_{m=1}^{M} E_{m} \lambda_{m} \exp \left(-\lambda_{m} s\right) \\
& +\sum_{l=1}^{L} A_{l} \frac{s^{l-1} \lambda_{e}^{l} \exp \left(-\lambda_{e} s\right)}{\Gamma(l)},
\end{aligned}
$$

where

$$
E_{m}=\left(\frac{\lambda_{e}}{\lambda_{e}-\lambda_{m}}\right)^{L} \prod_{u=1, u \neq m}^{M} \frac{\lambda_{u}}{\lambda_{u}-\lambda_{m}} .
$$

The coefficients $A_{l}$ can be found by solving a system of $L$ equations. The equation system is established by randomly choosing $L$ distinct values of $\lambda$ but not equal to $\lambda_{m}$ and $\lambda_{e}$. Denoting $L$ values of $\lambda$ as $B_{p}$ with $p=1,2, \ldots, L$.

$$
\begin{aligned}
& \left(\frac{\lambda_{e}}{\lambda_{e}-B_{p}}\right)^{L} \prod_{m=1}^{M} \frac{\lambda_{m}}{\lambda_{m}-B_{p}} \\
& \quad=\sum_{m=1}^{M} \frac{E_{m} \lambda_{m}}{\lambda_{m}-B_{p}}+\sum_{l=1}^{L} \frac{A_{l} \lambda_{e}}{\left(\lambda_{e}-B_{p}\right)^{l}} .
\end{aligned}
$$

$A_{l}$ is an element of matrix $A=C^{-1} D=\left[A_{1}, A_{2}, A_{L}\right]^{T}$. Where $C$ is an $L x L$ matrix with elements $C_{u v}=$ $\left(\left(\lambda_{e} /\left(\lambda_{e}-B_{u}\right)\right)^{v}\right.$ and $D=\left[D_{1}, D_{2}, D_{L}\right]^{T}$ with elements $D_{u}=\left(\lambda_{e} /\left(\lambda_{e}-B_{u}\right)\right)^{L} \prod_{m=1}^{M} \lambda_{m} /\left(\lambda_{m}-B_{u}\right)-$ $\sum_{m=1}^{M}\left(\lambda_{m} E_{m}\right) /\left(\lambda_{m}-B_{u}\right)$.

Next, the PDF of $z=x / y$ can be evaluated as [18, eq. (6-59)]:

$$
\begin{aligned}
f_{z}(z)= & \int_{\delta}^{\infty} y f_{x}(y z) f_{y}(y) d y \\
= & \lambda_{0} \exp \left(-\lambda_{0} z \delta\right) \sum_{m=1}^{M} \lambda_{m} E_{m} \Phi_{m}(z) \\
& +\sum_{l=1}^{L} \lambda_{e}^{l} A_{l} \Phi_{l}(z)
\end{aligned}
$$


Where:

$$
\begin{aligned}
& \Phi_{m}(z)=\frac{\delta}{\lambda_{0} z+\lambda_{m}}+\frac{1}{\left(\lambda_{0} z+\lambda_{m}\right)^{2}}, \\
& \Phi_{l}(z)=\frac{\delta}{\left(\lambda_{0} z+\lambda_{e}\right)^{l}}+\frac{l}{\left(\lambda_{0} z+\lambda_{e}\right)^{l+1}} .
\end{aligned}
$$

The CDF of $f_{z}(z)$ can be expressed as:

$$
\begin{aligned}
F_{z}(z)= & \int_{0}^{z} f_{z}(x) d x \\
= & \lambda_{0} \sum_{m=1}^{M} \lambda_{m} E_{m} \int_{0}^{z} \exp \left(-\lambda_{0} x \delta\right) \Phi_{m}(x) d x \\
& +\sum_{l=1}^{L} \lambda_{e}^{l} A_{l} \int_{0}^{z} \exp \left(-\lambda_{0} x \delta\right) \Phi_{l}(x) d x .
\end{aligned}
$$

Both integrals of Eq. 11 can be solved by substitution, hence:

$$
\begin{aligned}
F_{z}(z)= & \sum_{m=1}^{M} E_{m}\left(1-\frac{\lambda_{m} \exp \left(-\lambda_{0} z \delta\right)}{\lambda_{0} z+\lambda_{m}}\right) \\
& +\sum_{l=1}^{L} A_{l}\left[1-\frac{\lambda_{e}^{l} \exp \left(-\lambda_{0} z \delta\right)}{\left(\lambda_{0} z+\lambda_{e}\right)^{l}}\right] .
\end{aligned}
$$

Considering that the channel of each link in the network can be modelled by Rayleigh fading, we can assume that the sum representing interference in Eq. 2 is a sum of $M$ exponential random variables with a distinct mean $1 / \rho_{m}=$ $P_{C_{m}} d_{C_{m}}^{-\alpha}$ and $L$ exponential random variables with the same mean $1 / \rho_{e}=P_{C_{l}} d_{C_{l}}^{-\alpha}$ :

$$
\sum_{n=1}^{N}\left|h_{n R}\right|^{2} P_{C_{n}} d_{C_{n}}^{-\alpha}=\sum_{m=1}^{M} \frac{1}{\rho_{m}}\left|h_{m R}\right|^{2}+\sum_{l=1}^{L} \frac{1}{\rho_{e}}\left|h_{l R}\right|^{2} .
$$

Let $1 / \rho_{d}=P_{D} d_{D}^{-\alpha}$. Using Lemma 1 in Eq. 2 and inserting it in Eq. 3 we obtain the outage probability:

$$
\begin{aligned}
\operatorname{Pr}\left[\gamma_{D_{R}}<\right. & \left.\gamma_{0} \mid L_{C_{1}}, \ldots, L_{C_{n}}\right]=F_{\gamma_{D_{R}}}\left(\gamma_{0}\right) \\
= & \sum_{m=1}^{M} E_{m}\left(1-\frac{\rho_{m} \exp \left(-\rho_{d} \gamma_{0} N_{0}\right)}{\rho_{d} \gamma_{0}+\rho_{m}}\right) \\
& +\sum_{l=1}^{L} A_{l}\left[1-\frac{\rho_{e}^{l} \exp \left(-\rho_{d} \gamma_{0} N_{0}\right)}{\left(\rho_{d} \gamma_{0}+\rho_{e}\right)^{l}}\right] .
\end{aligned}
$$

\section{Outage performance analysis}

\subsection{DRC analysis}

One approach to interference mitigation is to use a distanceconstrained resource-sharing criterion (DRC) proposed in [14]. In this approach, the interferences from cellular users towards the D2D receiver (C2D) are controlled by the BS by selecting a resource sharing CUE, whose distance to D2D receiver meets predefined minimum distance requirements, further referenced as the DRC size. Although originally the DRC approach was proposed for a single cell scenario, it can be easily extended to a multi-cell case by using the DRC in each affected BS.

In this section, we present an analysis of the DRC approach in a scenario consisting of 19 hexagonal cells with ISD $S=500 \mathrm{~m}$ and hexagon edge length $R=S / \sqrt{3} \mathrm{~m}$. The power control scheme, for both the CUEs and DUEs, is the target SNR power control (TSPC), where the transmit power is selected to reach a fixed average SNR target $\sigma_{0}$, i.e., $P=$ $\sigma_{0} N_{0} r_{c}^{\alpha}$. Other relevant parameters are: $d_{D}=100 \mathrm{~m}, N_{0}=$ $-174 \mathrm{dBm} / \mathrm{Hz}, \alpha=4, \gamma_{0}=0 \mathrm{~dB}, P_{\max }=-46 \mathrm{dBm} / \mathrm{Hz}$ and bandwidth $B=1 \mathrm{MHz}$. This set of parameters represents a typical settings for a macro BS in LTE networks and is aligned with the values in [14].

Outage probability analysis in a multi-cell environment can be computationally demanding. To evaluate the average outage probability, we should consider all valid CUEs positions in each cell separately. Thus, the number of calculations in the case of the considered 19-cell scenario, and $1 \mathrm{~m}$ resolution, would be: $C=\left[(3 \sqrt{3} / 2)(R /(1[m]))^{2}\right]^{19}$. Therefore, we limited the simulations to $10^{5}$ independent random realizations of $L_{C_{n}}$ and limited the analysis area by considering only the cells, and the CUEs, that are located within a predefined circle-shaped search area centred at the location of the D2D receiver. The size of this area in these considerations was $2 R$. For a baseline we compared the multi-cell scenario to a single-cell case. All the simulations were carried out with a dedicated $\mathrm{C}++$ simulator.

We evaluated the outage probability for various locations of the D2D receiver. In the presented results, we assumed that the D2D receiver was located in the central cell, at the angle $\varphi_{D}=\pi / 4$ with variable radius $r_{D} \in\left(1, r_{D}^{\max }\right)$ where $r_{D}^{\max }=S /\left|\sin \left(\varphi_{D}\right)\right|+\sqrt{3}\left|\cos \left(\varphi_{D}\right)\right|$. From the analysis performed in [14], we know that the SNR target value of CUEs in TSPC scheme has a big impact on the outage probability performance of the $\mathrm{D} 2 \mathrm{D}$ receiver. Thus, the selection of the TSPC settings for the D2D transmitter and CUEs is not a trivial task. In the following analysis, based on the results of [14], we assumed that the SNR target is $\sigma_{C}=20 \mathrm{~dB}$ and $\sigma_{D}=40 \mathrm{~dB}$ for CUEs and the D2D transmitter, respectively. 
Fig. 2 D2D outage probability for different DRC sizes and DUE receiver positions: $r_{d}=0.3 r_{D}^{\max }, \ldots, 0.9 r_{D}^{\max }$ for a single cell scenario $(\mathrm{N}=1)$ and a multi-cell scenario $(\mathrm{N}=19)$

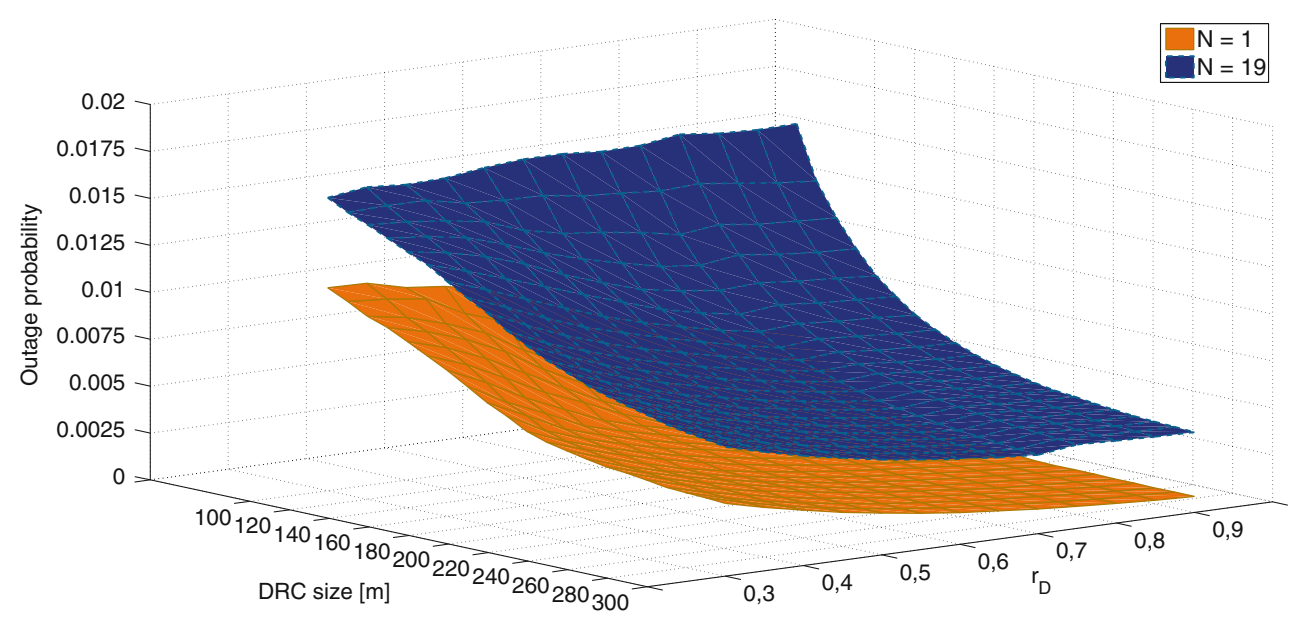

Figure 2 presents the outage performance of the D2D receiver against the size of the DRC region and the location of the D2D receiver. The results show that the DRC-based solution proves to be effective in reducing the outage probability in the FRF-1 systems. Considering the D2D receiver location, we can notice that the further away from the BS, the lower the outage probability. However, at a certain point the outage probability increases due to the increased impact of inter-cell interference. When comparing the outage performance of the FRF-1 scenario to the single cell scenario we notice a substantial increase in outage probability. Therefore, to enhance the performance of D2D communications in the FRF-1 system, a proper power control scheme should also be applied on top of the interference management solution such as DRC. In the next section, we propose and analyse a location-based power control mechanism for D2D communications.

\subsection{Location-based power control for D2D}

We propose a location-based target Signal-to-Interference Ratio (SIR) power control (LTSIPC) scheme for D2D communications. This power control mechanism utilizes users information about users locations to estimate the interference experienced by the D2D receiver. The SIR of the $\mathrm{D} 2 \mathrm{D}$ receiver is given by:

$\xi_{D_{R}}=\frac{\left|h_{D 21}\right|^{2} P_{D} d_{D}^{-\alpha}}{\sum_{n=1}^{N}\left|h_{n 1}\right|^{2} P_{C_{n}} d_{C_{n}}^{-\alpha}}$.

Based on this equation, the transmit power of the D2D transmitter can be derived by setting an SIR target $\xi_{0}$ :

$P_{D}=\frac{\xi_{0} \sum_{n=1}^{N}\left|h_{n 1}\right|^{2} P_{C_{n}} d_{C_{n}}^{-\alpha}}{\left|h_{D 21}\right|^{2} d_{D}^{-\alpha}}$.

Since we are using only location information to determine the interference, the knowledge on instantaneous channel coefficients $h_{D 21}$ and $h_{n 1}$ is not available to the power control mechanism. Therefore, to simplify (16), we assume that the mean value of both $\left|h_{D 21}\right|^{2}$ and $\left|h_{n 1}\right|^{2}$ is equal to 1 .

Fig. 3 CDF of DUE transmit power

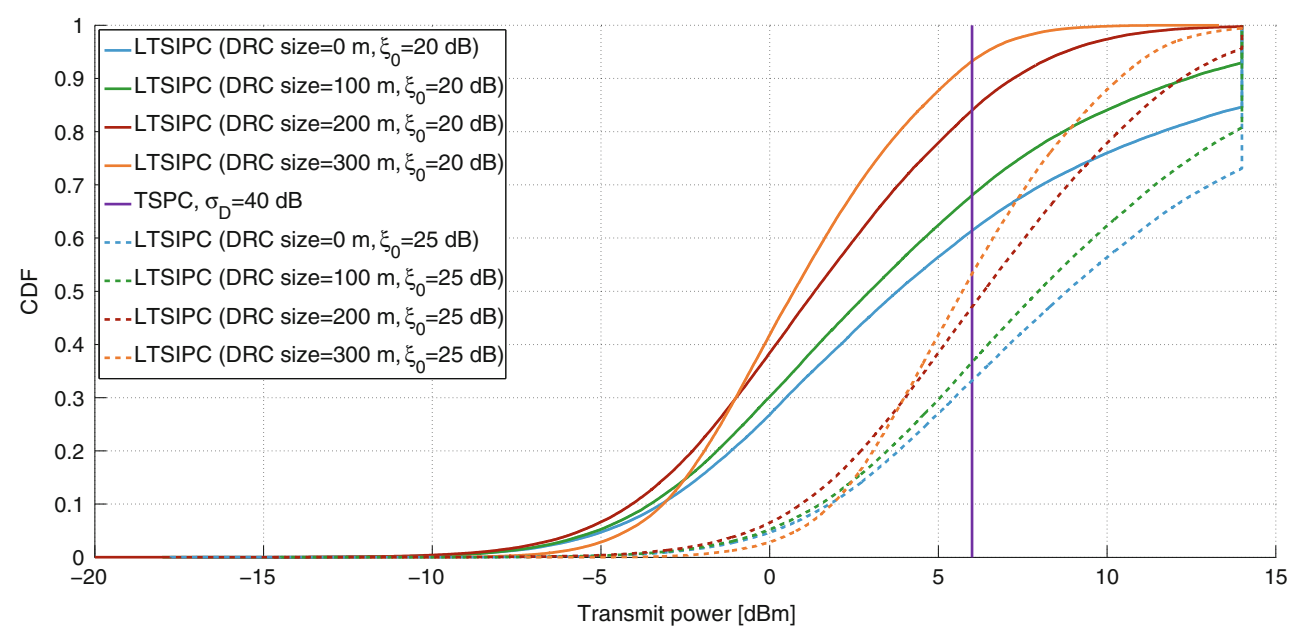


Fig. 4 D2D outage probability for different DRC sizes and power control mechanisms

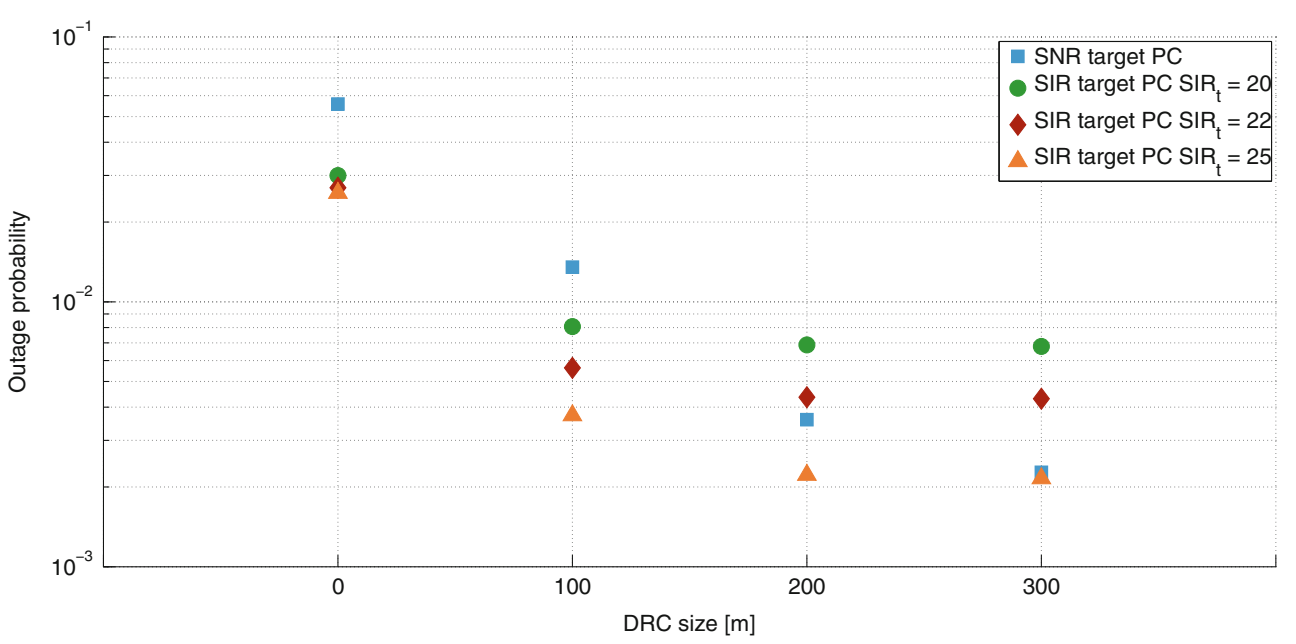

Taking this assumption into account, and setting the upper limit on transmit power $P_{\max }$, we obtain:

$P_{D}=\min \left(P_{\max }, \xi_{0} d_{D}^{\alpha} \sum_{n=1}^{N} P_{C_{n}} d_{C_{n}}^{-\alpha}\right)$.

The performance of the LTSIPC was evaluated in the same scenario as the DRC sharing user selection scheme, with additional assumptions that the location of the D2D receiver is fixed and close to the cell border, i.e., $\left(0.8 r_{D}^{\max }, \pi / 4\right)$ with respect to the central cell BS. Cellular users are using TSPC as a power control mechanism and the DUE transmitter is using LTSIPC.

We compared the LTSIPC with TSPC for various DRC sizes, and the results are presented in Fig. 3. The LTSIPC is more flexible than the TSPC and allows for a dynamic adjustment to the changes in the network. We can see that for $\xi_{0}=20 \mathrm{~dB}$ and no DRC scheme, the transmit power resulting from the LTSIPC is lower than the one resulting from TSPC in around $60 \%$ of situations. Increasing the $\xi_{0}$ value to $25 \mathrm{~dB}$ reduces this percentage to around $33 \%$. However, by applying the DRC mechanism on top of the LTSIPC, we can shift the CDF of the transmit power to lower values. For instance, for the DRC size of $100 \mathrm{~m}$ and $\xi_{0}=20 \mathrm{~dB}$, the resulting transmit power setting of the LTSIPC is lower than of the TSPC in around $70 \%$ of situations. By increasing the size of the DRC to $300 \mathrm{~m}$ this percentage can be increased to $93 \%$ of situations.

Finally, we compared the LTSIPC and TSPC in terms of outage performance. In Fig. 4, we present the outage performance against the DRC size and different $\xi_{0}$ values of the LTSIPC. We can see that the LTSIPC alone can reduce the outage probability. The joint application of the DRC user selection scheme and LTSIPC can further enhance the outage performance. The LTSIPC with $\xi_{0}=25 \mathrm{~dB}$ has the best outage performance for all considered DRC sizes. However, increasing the size of the DRC exclusion region has a greater impact on the outage probability in the TSPC scheme and for the case of a DRC radius of $200 \mathrm{~m}$, the TSPC outperforms the LTSIPC with $\xi_{0}$ lower than $25 \mathrm{~dB}$. Nevertheless, if we consider the fact that the transmit power of D2D communications is lower in $84 \%$ of situations in the case of $\operatorname{LTSIPC}\left(\xi_{0}=20 \mathrm{~dB}\right)$ compared to the TSPC, then we might still opt to use the LTSIPC for the reduced power benefit. Another aspect that has to be considered is the selection of the DRC size. By making the DRC exclusion area bigger, we decrease the number of potential cellular users for sharing, thus reducing the transmission opportunities of D2D communications. With this in mind, the LTSIPC has advantage over TSPC with a better outage performance for lower DRC sizes.

\section{Conclusion}

In this paper, we analysed the outage probability of D2D communications in a multi-cell FRF-1 system that shares the uplink cellular resources. We proposed a power control mechanism that, based on locations of the users and SIR target value, estimates the power setting for D2D communications. The results show that the D2D interference management in the FRF-1 network is not a trivial task. However, the location based approach for both selection of sharing CUEs and power control is a viable and simple solution to enable D2D communications in FRF-1 systems.

Open Access This article is distributed under the terms of the Creative Commons Attribution 4.0 International License (http:// creativecommons.org/licenses/by/4.0/), which permits unrestricted use, distribution, and reproduction in any medium, provided you give appropriate credit to the original author(s) and the source, provide a link to the Creative Commons license, and indicate if changes were made. 


\section{References}

1. Janis P, Yu C-H, Doppler K, Ribeiro C, Wijting C, Hugl K, Tirkkonen O, Koivunen V (2009) Device-to-device communication underlaying cellular communications systems. Int J Commun Network Syst Sci 2(15)

2. Seppala J, Koskela T, Chen T, Hakola S (2011) Network controlled Device-to-Device (D2D) and cluster multicast concept for LTE and LTE-A networks. In: Proceedings of IEEE wireless communications and networking conference (WCNC) 2011, pp 986-991

3. Doppler K, Rinne M, Wijting C, Riberio C, Hugl K (2009) Device-to-device communication as an underlay to LTE-advanced networks. IEEE Commun Mag 47:42-49

4. METIS project webpage. [Online]. Available: https://www. metis2020.com

5. Yu C-H, Doppler K, Ribeiro C, Tirkkonen O (2009) On the performance of device-to-device underlay communication with simple power control. In: Proceedings of IEEE vehicular technology conference

6. Yu C-H, Doppler K, Ribeiro C, Tirkkonen O (2009) Power optimization of device-to-device communication underlaying cellular communication systems. In: Proceedings of IEEE international conference on communications

7. Gu J, Bae SJ, Choi B-G, Chung MY (2011) Dynamic power control mechanism for interference coordination of device-to-device communication in cellular networks. In: Third international conference on ubiquitous and future networks (ICUFN'11), pp 71-75

8. Janis P, Koivunen V, Ribeiro C, Korhonen J, Doppler K, Hugl K (2009) Interference-aware resource allocation for device-to-device radio underlaying cellular networks. In: IEEE, 69th Vehicular Technology Conference Spring (VTC) Spring'09, pp 1-5

9. Zulhasnine M, Huang C, Srinivasan A (2010) Efficient resource allocation for device-to-device communication underlaying LTE network. In: IEEE 6th international conference on wireless and mobile computing networking and communications

10. Reider N, Fodor G (2012) A distributed power control and mode selection algorithm for D2D communications. EURASIP J Wireless Commun Netw 2012:266

11. Bao P, Yu G (2012) An interference management strategy for device-to-device underlaying cellular networks with partial location information. In: Proceedings of personal indoor and mobile radio communications (PIMRC'12), pp 465-470

12. Min H, Lee J, Park S, Hong D (2011) Capacity enhancement using an interference limited area for device-to-device uplink underlaying cellular networks. IEEE Trans Wirel Commun 10(12):39954000

13. Chen X, Chen L, Zeng M, Zhang X, Yang D (2012) Downlink resource allocation for Device-to-Device communication underlaying cellular networks. In: Proceedings of IEEE 23rd international symposium on personal indoor and mobile radio communications (PIMRC), pp 232-237

14. Wang H, Chu X (2012) Distance-constrained resource-sharing criteria for device-to-device communications underlaying cellular networks. IET Electron Lett 48(9):528-530

15. Wang H, Xia K, Chu X (2013) On the position-based resource sharing for device-to-device communications underlaying cellular networks. In: Proceedings of international conference on communications in China (ICCC,'13), pp 135-140

16. Chae HS, Gu J, Choi BG, Chung MY (2011) Radio resource allocation scheme for device-to-device communication in cellular networks using fractional frequency reuse. In: The 17th Asia Pacific Conference on Communications IEEE

17. Khuong HV, Kong H-Y (2006) General expression for pdf of sums of independent exponential random variables. IEEE Commun Lett 10(3):159-161

18. Papoulis A, Pillai SU (2002) Probability, random variables and stochastic process. McGraw-Hill 